\title{
Modeling sediment pulses on an armored channel
}

\author{
C. Juez, E. Battisacco, M.J. Franca \& A.J. Schleiss \\ Laboratory of Hydraulic Constructions, École Polytechnique Fédérale de Lausanne, Switzerland
}

\begin{abstract}
Dams trap sediments in the upstream reservoir, thus the continuity in sediment transport along the river is interrupted. Several negative aspects related to the lack of sediment supply were observed downstream many dams and the artificial replenishment of sediment was applied as a method to restore natural morphological conditions. The replenishment technique may be seen as a pulse of sediments added to the channel. Once the pulse-like volume of sediments is added into the reach, it is seen like an alteration of the channel geometry producing cross section narrowing and flow acceleration lengthwise the river. The river geometry modification together with the water flow allow erosion and transportation of the replenished material. In this work, the response of the flow to the material added in the channel is studied by means of the 2D shallow water equations model in combination with the Exner equation for the sediment continuity equation. Computational outcomes are compared with experimental data obtained from several replenishment configurations studied in the laboratory. It is observed how the bed fining provokes a larger spread of the material without aggradation within the armored channel.
\end{abstract}

\section{INTRODUCTION}

Rivers downstream of dams may have lack in sediment supply and suffer of bed deepening. The phenomenon is more evident during floods, when dams create an obstacle to natural sediment transport (Brandt 2000, Petts et al. 2005, Grant et al. 2013). Thus, morphological and ecological issues affect the downstream river. In detail, when the transport capacity exceeds the sediment supply the water becomes hungry enhancing the riverbed incision, the generation of an armored layer and the coarsening of the bed (Kondolf, 1997). Also the ecosystem along the river is negatively influenced by the lack in sediment transport. By consequences, the water quality is lower and a loss in the aquatic and riparian habitats are remarkable (Merz et al. 2006, Kantoush et al. 2010). The above-mentioned effects limit the possibilities for fish spawning.

For the mitigation of these negative consequences the sediment replenishment has emerged as a promising and challenging technique able to fine the riverbed, to increase bed mobility and to enrich morphological complexity. The available techniques for this sediment replenishment differ according the discharge, grain size, volume and geometry of replenishment considered (Kantoush et al. 2010, Ock et al. 2013). The sediment replenishment can be seen as a sediment pulse added to the channel (Sklar et al. 2009). This pulse-like addition of material at the beginning causes a perturbation in the bed level. The decreasing of this topographic perturbation occurs by erosion and it is described in
Kantoush et al. (2010). They showed that sediment deposits are eroded by a combination of lateral erosion on the placed sediment toe by hydraulic forces and subsequent mass failure by gravitational mechanism due to over-steepening of the banks. After mass failure, the collapsed mass is transported further downstream. This mass of sediments is responsible for the bed fining which afterwards, it causes a rapid transport of the replenishment material (Battisacco et al. 2015, Bösch et al. 2016). These results are obtained by a series of laboratory experiments performed at the Laboratory of Hydraulic Constructions at École Polytechnique Fédérale de Lausanne (EPFL), in Switzerland. Furthermore, the same tests are reproduced numerically in this work by using the $2 \mathrm{D}$ shallow water equations and the Exner equation. The spatial and temporal evolution of the hydrodynamic and morphodynamic models is solved by a synchronous treatment (Aricò et al. 2008), since there is a strong interaction between the flow and the replenishment material. The set of equations is computed by means of an explicit Finite Volume numerical scheme (Juez et al. 2014) where the numerical stability is controlled dynamically by an augmented CFL condition.

The paper is structured as follows: first the methodology to obtain the experimental data is described. Then the next two sections are devoted to briefly outline the mathematical model and the numerical scheme considered. The following section presents the results obtained with this study and their later discussion. Finally, the conclusions of the work are summarized. 


\section{EXPERIMENTAL SETUP}

The laboratory flume is $15 \mathrm{~m}$ long, $2.5 \mathrm{~m}$ wide and $0.7 \mathrm{~m}$ height. Two morphological identical channels were created. The width of channel bed is $0.4 \mathrm{~m}$, the bank slope is 2:3 (height: length) and the longitudinal slope is 0.015 (Figure 1). The discharge is controlled by a pump system spilling water from the inlet basin upstream throughout the outlet basin placed downstream. Furthermore, the discharge is indirectly determined by the replenishment submerge condition. The laboratory model has a 1:10 scale factor. The bed grain size distribution, used for creating the fixed bed and the banks, is representative of a typi$\mathrm{cal}$ alpine river. The replenishment volume are composed by a finer grain distribution which dimension varies from 3 to $8 \mathrm{~mm}$, as it is showed in Figure 2 .

The ecological need for spawning grounds, scaled to laboratory scale, are considered in the grain choice. In addition, the replenished sediment is added to the channel following the high-flow stockpile method (Ock et al. 2013) and colored in red in order to be distinguishable from the channel bed. The total duration of the experiment is three hours although the main differences are observed during the first two hours.

In this work, the influence of two different geometrical configurations are tested and analyzed: parallel (configuration A) and alternated (configuration B) (Figure 3). The four blocks of replenished sediments are equal in shape (trapezoidal) and volume $\left(\approx 0.006 \mathrm{~m}^{3}\right)$.

The discharge imposed, equal to $0.019 \mathrm{~m}^{3} / \mathrm{s}$, aims to reach two objectives: (i) to allow the mobilization of the replenishment material but without

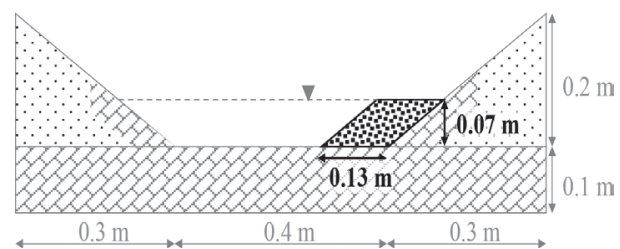

Figure 1. Schematic cross section of experimental channel with one replenishment volume (dark) (Bösch et al. 2016).

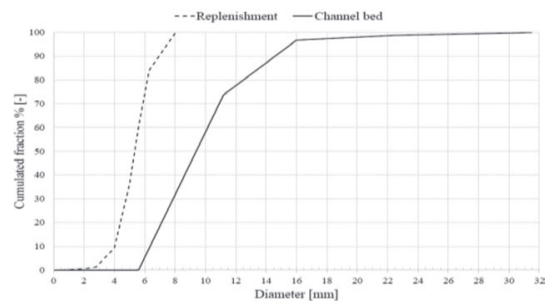

Figure 2. Grain size distribution for channel bed and replenishment material.
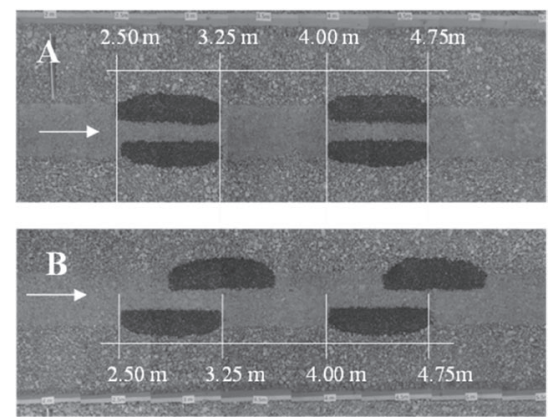

Figure 3. Experimental channel with replenished sediment (dark) for configuration A (top) and B (bottom) (Bösch et al. 2016).

eroding the bed channel or the banks and (ii) to achieve a $100 \%$ of submergence of the replenishments blocks (Battisaco et al. 2015).

Finally, data analysis was performed by means of photos taken at different time steps during the tests. A camera was installed on a carriage moving along the downstream and the traversal directions, and the photos were taken each $0.5 \mathrm{~m}$ along the channel and then merged together forming a panoramic view.

\section{MATHEMATICAL MODEL}

The mathematical model is based on the 2D shallow water equations, SWE, and the Exner equation. The SWE are derived from the Navier-Stokes equations by integrating the continuity and momentum equations over depth (Murillo et al. 2009). The resulting $2 \mathrm{D}$ system of equations is written in the conservative form as follows

$$
\frac{\partial \mathbf{U}}{\partial t}+\frac{\partial \mathbf{F}(\mathbf{U})}{\partial x}+\frac{\partial \mathbf{G}(\mathbf{U})}{\partial y}=\mathbf{T}_{\tau}+\mathbf{T}_{b}
$$

being

$$
\mathbf{U}=(h, h u, h v)^{T}
$$

the set of the conserved variables, with $h$ representing the water depth, $(u, v)$ are the depth averaged components of the velocity vector $\mathbf{u}$ along the $\times$ and $y$ coordinates respectively. The fluxes are given by

$$
\begin{aligned}
& \mathbf{F}=\left(h u, h u^{2}+\frac{1}{2} g h^{2}, h u v\right)^{T} \\
& \mathbf{G}=\left(h v, h u v, h v^{2}+\frac{1}{2} g h^{2}\right)^{T}
\end{aligned}
$$


where $g$ is the acceleration of the gravity. The source terms of the system take into account the gravity forces along the bottom and the frictional forces in the $x$ and $y$ direction of the horizontal plane. The shear stress is calculated by means of the Manning's law.

$$
\mathbf{T}_{\tau}=\left(0,-\frac{\tau_{b, x}}{\rho_{w}},-\frac{\tau_{b, y}}{\rho_{w}}\right)^{T} \quad \mathbf{T}_{b}=\left(0, \frac{p_{b, x}}{\rho_{w}}, \frac{p_{b, y}}{\rho_{w}}\right)^{T}
$$

System (1) is time dependent, non-linear, and contains source terms. Under the hypothesis of dominant advection it can be classified and numerically dealt with as belonging to the family of nonstrictly hyperbolic systems. The mathematical properties of (1) include the existence of a Jacobian matrix, $\boldsymbol{J n}$, of the flux normal to a direction given by the unit vector $\boldsymbol{n}$, defined as

$$
\mathbf{E n}=\mathbf{F} n_{x}+\mathbf{G} n_{y}
$$

whose components are

$$
\mathbf{J}_{n}=\left(\begin{array}{ccc}
0 & n_{x} & n_{y} \\
\left(g_{z} h-u^{2}\right) n_{x}-u v n_{y} & v n_{y}+2 u n_{x} & u n_{y} \\
\left(g_{z} h-v^{2}\right) n_{y}-u v n_{x} & v n_{x} & u n_{x}+2 v n_{y}
\end{array}\right)
$$

The eigenvalues of this Jacobian matrix are

$$
\lambda_{1}=\mathbf{u n}-c \quad \lambda_{2}=\mathbf{u n} \quad \lambda_{3}=\mathbf{u n}+c
$$

being $\mathrm{c}=(\mathrm{gh})^{1 / 2}$. These eigenvalues constitute the waves speed and provide information about directions in which the information travels.

On the other hand, the morphodynamic evolution of the replenishment of sediment is modeled by means of the Exner equation,

$$
\frac{\partial z}{\partial t}+\left(\frac{1}{1-p}\right) \frac{\partial q_{s, x}}{\partial x}+\left(\frac{1}{1-p}\right) \frac{\partial q_{s, y}}{\partial y}=0
$$

where $z$ is the bed level and $p$ is the porosity of the material. The terms qs, $x$ and qs,y are the solid sediment discharges in both directions. They are obtained through a solid discharge law, which in this work has been the Smart formula, Smart (1984).

Regarding equation (8), although it is not an hyperbolic equation a numerical estimation of the bed celerity can be computed by means of the sediment flux, normal to a direction given by the unit vector, $\lambda_{s} \approx\left(\frac{1}{1-p}\right) \frac{\partial \mathbf{q}_{s \mathbf{n}}}{\partial z}$

The current computation of this bed wave speed constitutes the basis of the upwind numerical discretization that will be defined in the next section.

\section{FINITE VOLUME MODEL}

\section{$4.1 \quad$ Numerical scheme}

For the sake of brevity the numerical scheme associated to the hydrodynamic model is not described. Full details can be found in Murillo et al. 2010. It consist of an explicit finite volume scheme based on an Augmented Roe solver, where the stability criteria is governed by the CFL condition given by the water waves speeds.

With respect to the numerical scheme for morphodynamic model, equation (8) is integrated over a fixed volume or grid cell $\Omega$, using the Gauss theorem:

$$
\frac{\partial}{\partial t} \int_{\Omega_{i}} z d \Omega+\oint_{\partial \Omega}\left(\frac{1}{1-p}\right)\left(\vec{\nabla} \mathbf{q}_{s}\right) d l=0
$$

Using Gauss theorem, assuming a piecewise representation of the variable $z$ and that the second integral can be written as the sum of fluxes across the cell edges,

$$
\frac{\partial}{\partial t} \int_{\Omega_{i}} z d \Omega+\sum_{k=1}^{N E}\left(\frac{1}{1-p}\right) \int_{l_{k}} \mathbf{q}_{s \mathbf{n}, k} d l_{k}=0
$$

Then, the Godunov first order method is built through a flux scheme, considering outcoming and incoming fluxes through the edges of the cell. Hence the bed level is updated as

$$
z_{i}^{n+1}=z_{i}^{n}-\sum_{k=1}^{N E}\left(\frac{1}{1-p}\right) \mathbf{q}_{s \mathrm{n}, k}^{*} \frac{\Delta t l_{k}}{A_{i}}
$$

where $A i$ is the area of the cell and $l k$ is the length of each wall of each cell and also,

$$
\mathbf{q}_{s \mathbf{n}, k}^{*}=\left\{\begin{array}{lll}
\mathbf{q}_{s \mathbf{n}, i} & \text { if } & \tilde{\lambda}_{s \mathbf{n}, k}>0 \\
\mathbf{q}_{\mathbf{s n}, j} & \text { if } & \tilde{\lambda}_{\mathbf{s n}, k}<0
\end{array}\right.
$$

Being $\mathrm{q}_{\mathrm{sn}, \mathrm{i}}$ and $\mathrm{q}_{\mathrm{sn}, \mathrm{j}}$ the bed load discharge computed between neighboring cells, $i$ and $j$, and the bed celerity is estimated as 


$$
\tilde{\lambda}_{s}=\left(\frac{1}{1-p}\right) \frac{\delta\left(\mathbf{q}_{s \mathrm{n}, k}\right)}{\delta z}
$$

with $\delta z=\mathrm{z}_{\mathrm{j}}-\mathrm{z}_{\mathrm{i}}$ and $\delta \mathrm{q}_{\mathrm{sn}, \mathrm{k}}=\mathrm{q}_{\mathrm{sn}, \mathrm{i}}-\mathrm{q}_{\mathrm{sn}, \mathrm{i}}$ the normal solid flux crossing neighboring cells sharing edge $\mathrm{k}$.

The linearization of the bed celerity in cases of flat bottom lead to unphysical values. This problem is bypassed by approximating the bed slope by the friction slope when calculating $\delta z$. More details can be found in Juez et al. 2014.

\subsection{Stability condition}

As it was stated in Leveque, 2002, the updated conserved variables are defined through the fluxes obtained within each cell, so, the computational time step, $\Delta t$, has to be chosen small enough for ensuring a stability region. Traditionally, the numerical stability has been controlled through a hydrodynamic dimensionless parameter, CFL,

$$
\Delta t \leq C F L \Delta t^{\lambda} \quad \Delta t^{\lambda}=\frac{\min \left(\chi_{i}, \chi_{j}\right)}{\max \left|\tilde{\lambda}_{m}\right|}
$$

Being $\chi$ the relevant distance, that following Murillo et al. 2010 in a 2D framework is defined as

$$
\chi_{i}=\frac{A_{i}}{\max _{k=1, N E} l_{k}}
$$

And $\tilde{\lambda}_{m}$ the hydrodynamic wave speeds. In addition, CFL $\leq 1 / 2$ in the case of triangular unstructured grids.

On the other hand, the bed celerity $\tilde{\lambda}_{\text {s }}$ from (14) has to be included in the stability control. Therefore, the overall restriction for the weakly-coupled system, hydrodynamic and morphodynamic, is revisited as

$$
\Delta t=C F L \frac{\min (\chi)}{\max \left|\tilde{\lambda}_{m}, \tilde{\lambda}_{s}\right|} \text { with } \quad C F L \leq 0.5
$$

\subsection{Geomorphological collapse}

In realistic transient geomorphological flows, the lateral erosion and sliding of the river banks play an important role on the channel changes. This effect needs to be modeled to reproduce correctly bed geometry evolution in combination with flow action. In this work, and following Murillo et al. $2010 \mathrm{~b}$, the effect of the geomorphological collapse of the replenishment of sediments is introduced in the simulation by a simple mass conservative mechanism of slope sliding failure, assuming that the angle of repose of submerged material of the bed can be approximated by the friction angle. The failure mechanism is applied by comparison between the bed slope in each cell edge and the angle of repose of saturated bed material.

\subsection{Bed fining}

he pulse of sediments added to the channel by means of the replenishment technique causes secondary effects. Due to the finer nature of the sediments of the replenishment, the initially coarser non-erodible channel suffers a change in the surface roughness, i.e. the sediments coming from the replenishment build a new layer over which the water column exerts a new friction value (Ferguson et al. 2015, Ferreira et al. 2012). In order to take into account this important characteristic, the Manning parameter, $n$, is continuously updated during the simulation as follows

$$
n=\left\{\begin{array}{lll}
\frac{1}{26}\left(d_{90}^{C}\right)^{1 / 6} & \text { if } & D D \leq d_{90}^{C}+d_{90}^{R} \\
\frac{1}{26}\left(d_{90}^{R}\right)^{1 / 6} & \text { if } & D D>d_{90}^{C}+d_{90}^{R}
\end{array}\right.
$$

where $\mathrm{d}_{90}$ and $\mathrm{d}_{90}$ are the non-erodible channel and the replenishment material respectively, and $\mathrm{DD}$ is the deposition depth of the added material for replenishment purposes.

\section{RESULTS}

\subsection{Comparison with experimental data}

Numerical results are herein compared with the experimental data obtained in the laboratory. The results are compared in terms of: (i) 2D temporal evolution of the material spreading through the covered surface, (ii) occupation ratio along the channel which is the area percentage that is covered by the replenishment material, (iii) travel distance of the center of the pulse mass and (iv) effect of the bed fining in the bed shear stress.

\subsection{Results visualization}

The 2D temporal evolution of the covered surface occupied by the replenishment material during the experiment is displayed in Figure 4 and Figure 5 (top views). Visual observations during the experiment show that the erosion of the blocks of replenishment occurs as a combination of fluvial erosion at the deposits toe and mass failure of the above 


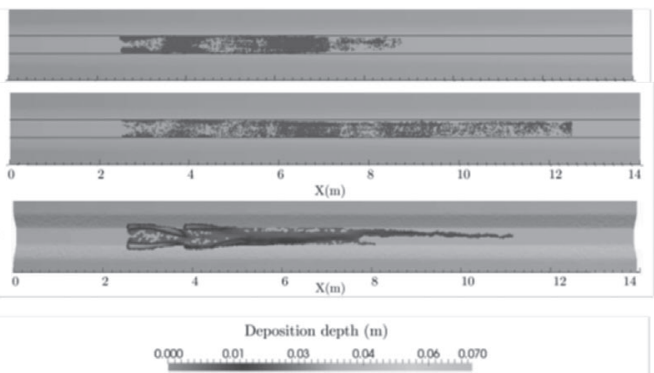

Figure 4. Configuration A (see Figure 3). 2D experimental data of the covered surface (top views) and 3D computed contour views simulated numerically (bottom views) for the deposition depth along the channel at times $\mathrm{t}=10$ and $120 \mathrm{~min}$.
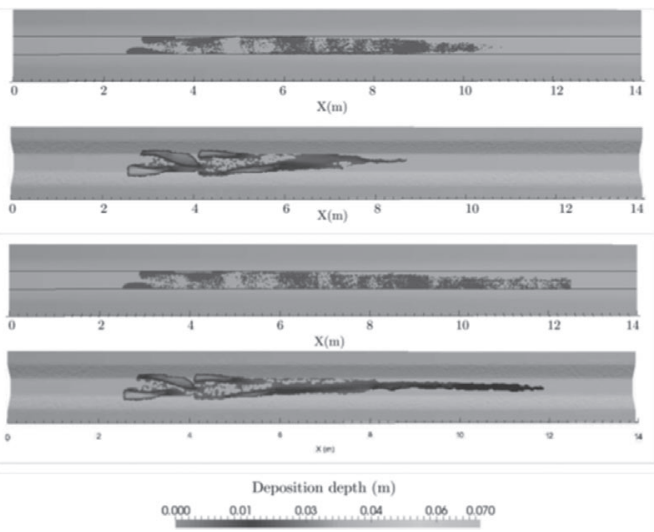

Figure 5. Configuration B (see Figure 3). 2D experimental data of the covered surface (top views) and 3D computed contour views simulated numerically (bottom views) for the deposition depth along the channel at times $\mathrm{t}=10$ and $120 \mathrm{~min}$.

placed sediment due to over-steepening. The collapsed mass is then further eroded and transported downstream by the flow. All these trends are captured by the numerical scheme.

When comparing the results in configuration A and $\mathrm{B}$, it is showed how the placement of the blocs plays an important role in the general behavior. The alternate configuration increases the erosion rate and enhances the creation of a bed form pattern downstream.

\subsection{Occupation ratio}

An important parameter when dealing with replenishment techniques is the occupation ratio (OR). This parameter is based on a $2 \mathrm{D}$ analysis and it shows the fraction of area that is covered by the replenishment material:
$O R=\frac{A_{R}}{A_{T}}$

where $A_{R}$ is the area which is occupied by the replenishment material and $A_{T}$ is the total area under analysis. This parameter has been computed for traversal slices of the channel with $0.1 \mathrm{~m}$ length. The OR aims at identifying the level of material aggregation on the channel along the longitudinal direction. For an adequate sediment replenishment process a dispersion behavior of the added material is required to achieve the most common goals to improve rivers ecology, such as fining bed grain size and enhanced bed elevation variations in order to improve rivers ecology. Hence, the OR quantifies the dispersion of the material of the replenishment along the channel.

The results for both configurations are displayed in Figure 6 and Figure 7. Regarding the experimental data it is observed a wider variety of peaks with configuration $\mathrm{B}$ due to its greater complexity. On the other hand, the general trend of the experimental data is well tracked by the numerical predictions since the maximum spreading in both configurations is achieved. However, it is pointed out that the richness in bed patterns is not reached by the numerical predictions. This can be justified by the fact that the peak in OR found in some areas are composed only by a few layers of material. Such level of accuracy suppose a challenge for any continuum approach as the one proposed in this work.

\subsection{Travel distance}

Travel distance (TD) of the center of mass is another important parameter which allows to verify the geomorphological response of the channel bed to gravel augmentation under given geometric and hydrodynamic conditions. The higher the TD value is, the bigger the impact of the replenishment is in the downstream direction. Furthermore, the analysis of the TD over time allows to estimate the propagation velocity of the sediment transport. The slope in the temporal evolution of transport distance can be seen as a transport velocity, where steep slopes indicate a fast movement. Furthermore, the position of the center of mass can be estimated for the entire deposition region observed or partially for several percentiles. Hence, $\mathrm{TD}_{\mathrm{X}}$ is the position where the $\mathrm{x} \%$ of the covered surface is located downstream. Figure 7 displays the travel distance for several percentiles of the covered surface for the computational results and the experimental data. Transport velocity for $\mathrm{TD}_{75}$ and $\mathrm{TD}_{95}$ are higher than of $\mathrm{TD}_{50}$ and $\mathrm{TD}_{25}$. Each percentile travels quicker during the first minutes; afterward, the transport velocity tends to slow down until an equilibrium stage is reached. 

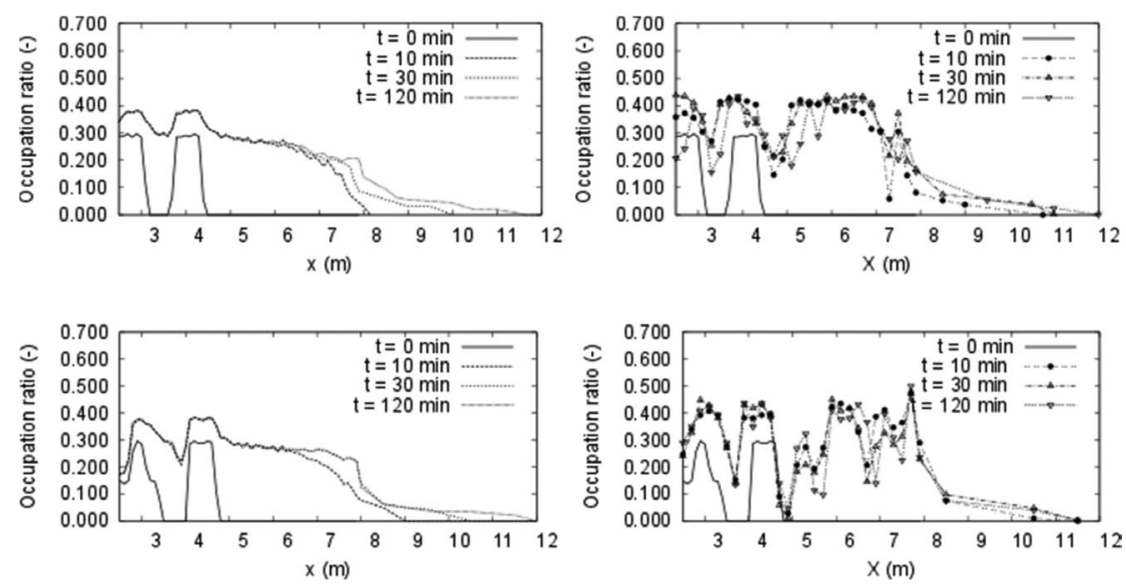

Figure 6. Temporal evolution of the occupation ratio (OR) for configuration A (top) and configuration B (bottom): numerical outcomes (left) and experimental data (right). Configuration according Figure 3.
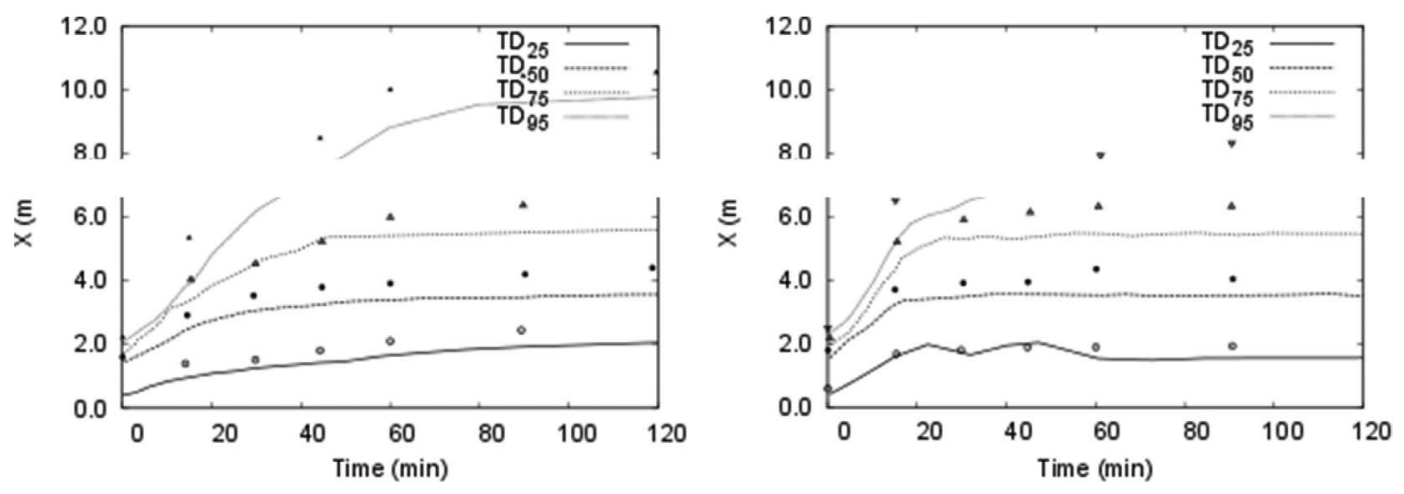

Figure 7. Temporal evolution of the travel distance (TD) for several percentiles for configuration A (left) and configuration $\mathrm{B}$ (right) obtained with numerical outcomes (continuous lines) and with experimental data (points). Configuration according to Figure 3.

In addition, the sediments in configuration $\mathrm{A}$ with the parallel blocks travels further mainly due to the effect of bed fining.

Regarding the numerical-experimental comparison, there is an accurate agreement between both results, although the experimental travel distance is always above the computational prediction. This is justified by the fact that the maximum run-out with the laboratory data is slightly larger than the one obtained with the numerical tool.

\subsection{Bed fining}

Bed fining explains the further distances travelled by configuration $\mathrm{B}$ since in that case the Occupation ratio is higher, i.e. more surface of the nonerodible channel is occupied by the replenishment material. The decrease of roughness during the replenishment results in higher shear stress, which leads to increase transport capacity. This explanation is also supported by a recent work, Ferguson et al. 2015, where they showed that bed fining and consequently increased shear stress raised transport capacity by an order of magnitude. Thus, in order to well reproduce this phenomenon when numerical modelling it is necessary to enrich the selection of the roughness coefficient by a dynamic selection as it has been performed in this work.

\section{CONCLUSIONS}

A work on 2D unsteady numerical modeling of a pulse of sediments added to a non-erodible chan- 
nel for replenishment purposes has been described. The numerical scheme solves a weak coupled model which includes the 2D SWE and the 2D Exner sediment continuity equation. It is written under the form of finite volumes and takes advantage of the upwind philosophy. The explicit scheme has shown robust stability, always controlled by an augmented CFL condition.

The computed results have been compared against experimental data. On the basis of the mathematical model, the erosion/deposition rate is calculated by means of the Smart formula and a dynamic roughness coefficient has been considered.

The results show a physical insight behavior, since the maximum spreading, occupation ratio and travel distance has been well reproduced. The effect of the bed fining due to the sediments added to the channel are also faced up by the results. Moreover no numerical instabilities appear despite the important interaction between the water and solid fluxes.

Further research needs to be devoted to explore the influence of a non-fixed channel bed where the vertical sorting among particles can play an important role on the overall behavior of the replenishment technique.

\section{ACKNOWLEDGEMENT}

This work was funded by the ITN-Programme (Marie Curie Actions) of the European Union's Seventh Framework Programme FP7-PEOPLE2013-ITN under REA grant agreement n_607394SEDITRANS. The sediment replenishment experiments were funded by FOEN (Federal Office for the Environment, Switzerland).

\section{REFERENCES}

Aricò, C. \& Tucciarelli, T. 2008. Diffusive modeling of aggradation and degradation in artificial channels. Journal of Hydraulic Engineering 134 (8): 1079-1088.

Battisacco, E., Maire, A., Franca, M.J. \& Schleiss, A.J. 2015. Physical modeling of artificial river replenishment techniques to restore morphological conditions downstream of dams. Proceedings of the 36th IAHR World Congress, Delft.

Bösch, L., Battisacco, E., A., Franca, M.J. \& Schleiss, A.J. 2016. Influence of consecutive sediment replenishment on channel bed morphology. Proceedings of River Flow 2016, St Louis.
Brandt, S.A. 2000. Classification of geomorphological effects downstream of dams. Catena 40 (4):375-401.

Ferreira, R.M.L., Franca, M., Leal, J.G.A.B \& Cardoso, A.H. 2012. Flow over rough mobile beds: friction factor and vertical distribution of the longitudinal mean velocity. Advances in Water Resources. 48: W05529.

Ferguson, R.I., Church, M. Rennie, C.D. \& Venditti, J.G. 2015. Reconstructing a sediment pulse: modeling the effect of a placer mining on Fraser River, Canada. Journal of Geophysical Research: Earth Surface. 120: 1436-1454.

Grant G.E, Schmidt J.C. \& Lewis S.L. 2013. A geological framework for interpreting downstream effects of dams on rivers. A peculiar river, American Geophysical Union: 203-219.

Juez, C., Murillo, J. \& García-Navarro, P. 2014. A 2D weakly-coupled and efficient numerical model for transient shallow flow and movable bed. Advances in Water Resources 71(1): 93-109.

Kantoush, S.A., Sumi, T., Suzuki, T. \& Murasaki, M. Impacts of sediment flushing on channel evolution and morphological processes: Case study of the Kurobe River, Japan. RiverFlow 2010, International Conference on Fluvial Hydraulic, 2010

Kondolf, G.M. 1997. Hungry water: effects of dams and gravel mining on river channels. Environmental Management,

Leveque, R.J. Finite Volume Methods for Hyperbolic Problems, Cambridge Univeristy Press, New York, 2002.

Merz, J., Pasternackc, G. \& Wheaton, J. 2006. Sediment budget for salmonid spawning habitat rehabilitation in a regulated river. Geomorphology 76(1): 207-228.

Murillo, J. \& García-Navarro, P., 2010. Weak solutions for partial differential equations with source terms: application to the shallow water equations, Journal of Computational Physics. 229, 4327-4368.

Murillo, J. \& García-Navarro, P., 2010b. An Exner-based coupled model for two-dimensional transient flow over erodible bed, Journal of Computational Physics. 229, 8704-8732.

Ock, G., Sumi, T. \& Takemon, Y. 2013. Sediment replenishment to downstream reaches below dams: implementation perspectives. Hydrological Research Letters 7(3): 54-59.

Petts G.E \& Gurnell A.M. 2005. Dams and geomorphology: research progress and future directions. Geomorphology 71(1-2): 27-47.

Sklar, L.S., Fadde, J., Venditti, J.G., Nelson, P.A., Wydzga, M.A., Cui, Y. \& Dietrich, W.E. 2009. Translation and dispersion of sediment pulses in flume experiments simulating gravel augmentation below dams. Water Resources Research 45(8): 1-14.

Smart, G., 1984. Sediment transport formula for steep channels, Journal of Hydraulic Engineering. 3, 267-276. 